\title{
A new approach for reliability-centered maintenance programs in electric power distribution systems based on a multiobjective genetic algorithm
}

\author{
Diego Piasson ${ }^{\mathrm{a}, *}$, André A.P. Bíscaro ${ }^{\mathrm{b}}$, Fábio B. Leão ${ }^{\mathrm{c}}$, José Roberto Sanches Mantovani ${ }^{\mathrm{c}}$ \\ a Technology Center of Mato Grosso, Mato Grosso State University, Barra do Bugres, Mato Grosso, Brazil \\ b Electrical Engineering Department, Mato Grosso State University, Sinop, Mato Grosso, Brazil \\ ${ }^{\mathrm{c}}$ Electrical Engineering Department, São Paulo State University - UNESP, Ilha Solteira, São Paulo, Brazil
}

\section{A R T I C L E I N F O}

\section{Article history:}

Received 23 November 2015

Received in revised form 22 March 2016

Accepted 23 March 2016

\section{Keywords:}

Fuzzy systems

NSGA-II

Power quality

Power system reliability

Preventive maintenance

Reliability centered maintenance

\begin{abstract}
A B S T R A C T
This paper proposes a multiobjective model to solve the mathematical problem of optimizing reliabilitycentered maintenance planning of an electric power distribution system (EPDS). The main goal is to minimize the preventive maintenance costs while maximizing the index of reliability of the whole system. In the proposed model, the limits of the indices, such as SAIDI and SAIFI, are considered as constraints of the maintenance programs. The reliability indices of the EPDS components are evaluated and updated by a fuzzy inference system. A NSGA-II algorithm was proposed to solve the multiobjective model that provides an optimized Pareto frontier. The results obtained from applying the proposed methodology to a system with three feeders and 733 components are presented, showing its robustness and quality for maintenance planning in EPDS.
\end{abstract}

(ㄷ) 2016 Elsevier B.V. All rights reserved.

\section{Contents}

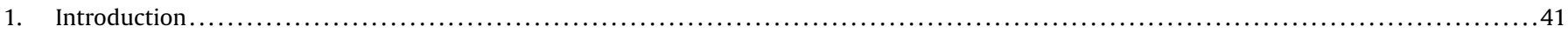

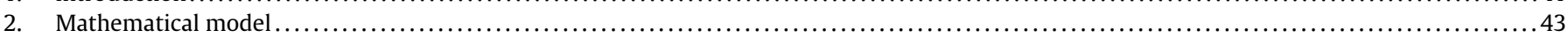

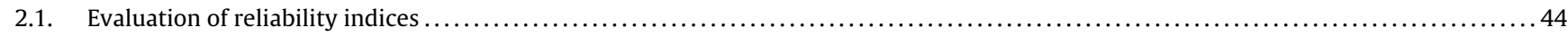

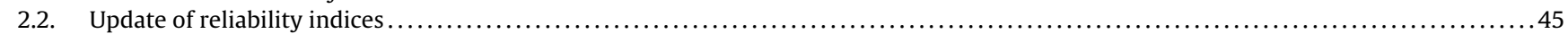

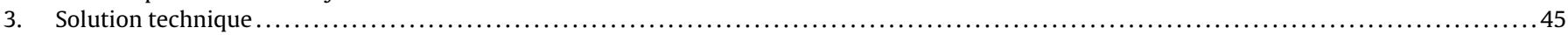

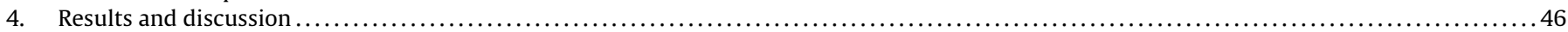

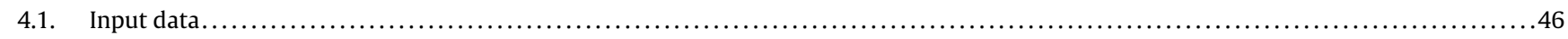

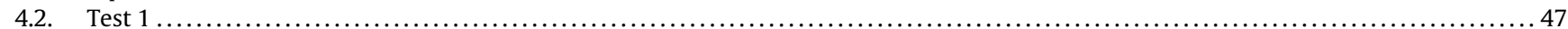

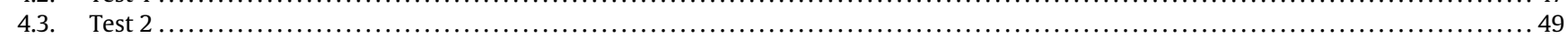

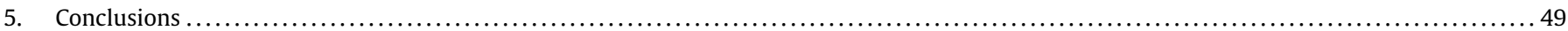

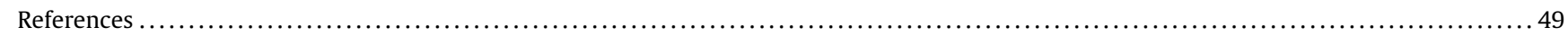

\section{Introduction}

Electric power distribution utilities must offer power supply services that have quality, appropriate voltage levels and a low interruption rate. To achieve these goals, specifically quality,

\footnotetext{
* Corresponding author. Tel.: +55 6596019058.

E-mail addresses: diegopiasson@yahoo.com.br, diegopiasson@gmail.com (D. Piasson).
}

regulatory agencies establish power quality indicators for supply services as well as targets and thresholds to be achieved by electric utilities. These companies usually carry out preventive maintenance (PM) programs to improve system reliability by establishing better working conditions to extend the useful life of their equipment $[1,2,22]$.

Distribution system reliability is one of the most important indices for evaluating the service quality of electric power distribution companies [3]. In particular, for a given distribution system and for each year of the regulatory period, regulatory agencies specify 


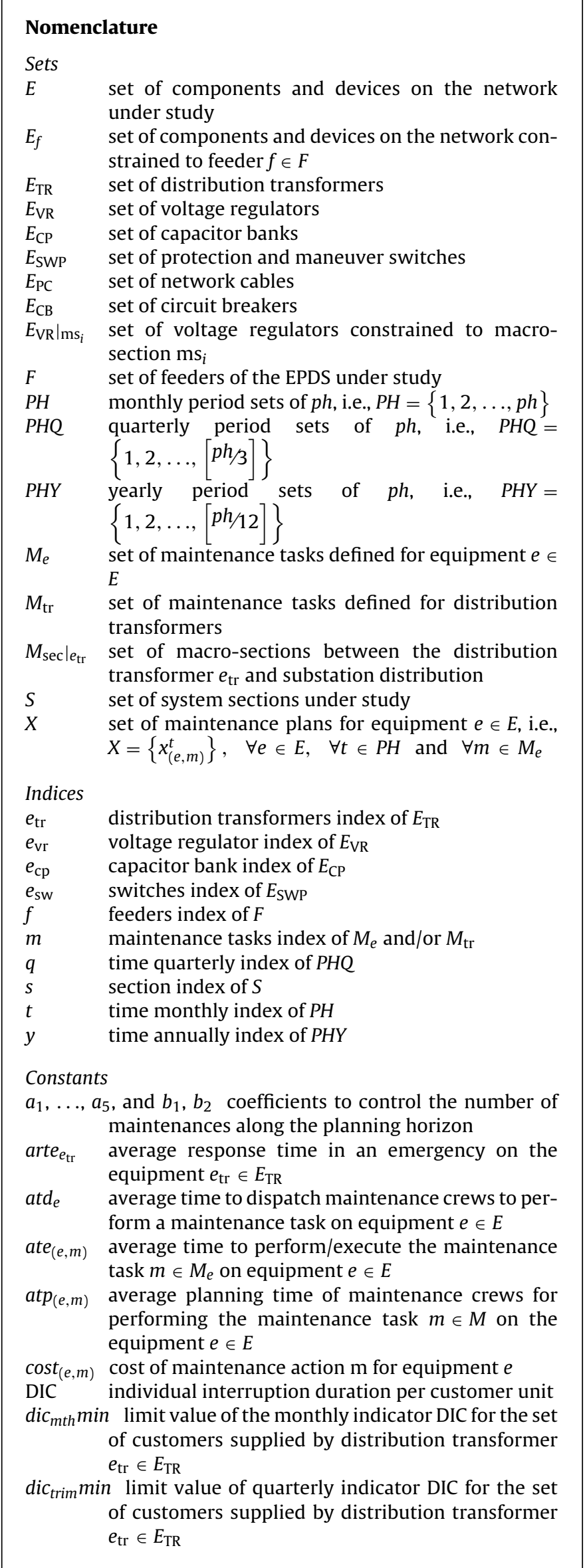

$d i c_{\text {year }} \min$ limit value of yearly indicator DIC for the set of customers supplied by distribution transformer $e_{\mathrm{tr}} \in E_{\mathrm{TR}}$

FIC interruption frequency individual per customer unit

fic $c_{m t h}$ min limit value of monthly indicator FIC for the set of customers supplied by distribution transformer $e_{\mathrm{tr}} \in E_{\mathrm{TR}}$

fic $c_{\text {trim }}$ min limit value of quarterly indicator FIC for the set of customers supplied by distribution transformer $e_{\mathrm{tr}} \in E_{\mathrm{TR}}$

fic yearmin limit value of yearly indicator FIC for the set of customers supplied by distribution transformer $e_{\text {tr }} \in$ $E_{\mathrm{TR}}$

$j \quad$ monthly update rate of the cost of maintenance task $\max \left\{m \in M_{e}\right\}$ the highest level of maintenance defined for equipment $e \in E$

ph planning horizon

$t_{\text {feasible }_{f}}$ time of maintenance teams (in hours) to perform scheduled maintenance tasks

$u r_{\max }$ maximum supported value for the failure probability (Unreliability) of capacitor banks

$w_{s} \quad$ active power loads fed by section $s \in S$

\section{Fuzzy outputs}

$C_{E_{\mathrm{PC}}}^{m s_{i}}(t)$ reliability of network cables set of the macro-section $m s_{i}$, with $i \in M_{\text {sec }} e_{\text {tr }}$ at time $t$

$C_{e_{\mathrm{SW}}}^{m s_{i}}(t) \quad$ reliability of protection switch $e_{\mathrm{sw}} \in E_{\mathrm{SWP}}$ of macrosection $m s_{i}$, contained in $M_{\text {sec }} e_{\text {tr }}$ at time $t$

$C_{e}(t) \quad$ reliability of equipment $e \in E$ at time $t$

$C_{e_{\mathrm{rt}}}^{m s_{i}}(t)$ reliability of voltage regulator $e_{\mathrm{rt}} \in E_{\mathrm{RT}}$ constrained to macro-section $\left.m s_{i} \in M_{\mathrm{sec}}\right|_{e_{\mathrm{tr}}}$ at time $t$

$C_{e_{\mathrm{bc}}}(t)$ reliability of capacitor banks at time $t$

$C_{e_{\mathrm{Vr}}}(t)$ reliability of voltage regulator $e_{\mathrm{Vr}} \in E_{\mathrm{VR}}$ at time $t$

$C_{e_{\mathrm{tr}}}(t) \quad$ reliability of distribution transformer $e_{\mathrm{tr}} \in E_{\mathrm{TR}}$

$C_{S}(t) \quad$ reliability of section $s \in S$ at time $t$

\section{Variables}

$a_{e}^{t} \quad$ apparent age of equipment $e \in E$ at time $t$

$P(t) \quad$ failure probability at $t \in P H$

$x_{(e, m)}^{t} \quad$ binary decision variable to perform (or not) the maintenance task $m$ at time $t$ on the equipment $e$

the values of these indices based on historical values. In this scenario, the planning operation department (POD) of the utilities can propose different strategies to improve the reliability performance of their distribution systems. Some actions commonly adopted are network maintenance actions to prevent fault events, introduction/reinforcement of control and automation devices, adoption of new network management paradigms (island mode operation in the restorative state), and reallocation or installation of additional switches along the distribution system. However, achieving this goal may involve an increase in planning and operating costs. With the competitiveness of the electricity market, a reduction in the operational and investment costs of the systems is required, which therefore requires reducing the costs of PM programs [27]. These costs constitute the largest expense for companies in this sector [2]. Thus, the optimization of PM programs has become critical for distribution companies to fulfill these two conflicting objectives, i.e., reducing the operational and investment costs and improving the electric power distribution reliability. In this context, reliability centered maintenance (RCM) is presented as an efficient methodology to relate equipment maintenance with system reliability $[4,5]$. 
A large number of methods for PM optimization in power systems focusing on RCM can be found in literatures [6-8]; some works focused on component maintenance in a medium voltage EPDS network [2,3,9-14]. In these studies, the main differences are related to: (1) the number and nature of objective functions considered in the model; (2) the decision variables considered during modelling; (3) the methodology for assessing the reliability index of each piece of equipment; (4) the types of maintenance that can be scheduled; (5) the methodology for assessing the impact of maintenance on reliability; and (6) the technique for solving the model. The following works are briefly described to highlight the aforementioned aspects.

Reference [9] proposes a binary programming model to identify the optimal maintenance level for each system component. The goal is to minimize the System Average Interruption Frequency Index (SAIFI) of a main feeder. The model constraints are the availability of financial and human resources to perform maintenance tasks. Different levels of maintenance and failure rates are considered for each feeder branch. The model is tested using a fictitious feeder. In the first test, a constant failure rate is considered, and in the second test, these rates and the impact factor of maintenance tasks in each element of the network are modelled as fuzzy sets.

Reference [2] proposes a heuristic method for comparing the effect of different maintenance tasks on the reliability of an EPDS. Equipment failure rates are considered in two ways: constant and varying as a function of time and maintenance measures. The impact of each maintenance task in the network is evaluated through the variation produced in a cost function that considers the costs of failures, PM and interruptions. The relationship between PM tasks and the reliability of system components is not a straightforward relationship. The methodology is tested using an $11 \mathrm{kV}$ system composed of underground cables. The planning horizon considered is 15 years, and the maintenance tasks are allocated annually.

References [10,15] propose a multiobjective optimization model for the EPDS maintenance problem. This model considers the expected yearly customer interruption cost and the maintenance cost of the network (both corrective and preventive). However, the objective functions are combined into a single function. The power quality indicators imposed by regulatory agencies are not considered in the modelling. Furthermore, the study does not extend the analysis of failure behavior over time and with maintenance measures. Equipment failure rates are considered to be constant. The connection between component and system reliability is assessed using an index that corresponds to the expected total customer interruption cost caused per component over a yearly time interval. Three maintenance measures for each component are adopted, namely: maintain the current PM level; improve the PM level; and decrease the PM level. Multipliers were utilized to change the failure rates of the components as a function of the maintenance tasks that were performed. The impact of each maintenance task on maintaining the reliability of the components was also not fully analyzed. The model was tested on a system composed of $178 \mathrm{com}-$ ponents, including circuit breakers, cables, transformers, busbars and fuses. A Metaheuristic Particle Swarm method was used to solve the proposed model.

References $[13,14]$ propose a practical framework for implementing the RCM procedure in EPDS. In the methodology proposed, a heuristic procedure is used for choosing optimal maintenance strategies that satisfy predetermined desired targets. A weighting table is used for failure rate modelling as in [11]. The maintenance strategies are prioritized based on the benefit-cost ratio, and they are centered on expert's experiences and operator's knowledge. In $[10,15]$, restrictions involving quality indicators imposed by regulatory agencies, such as System Average Interruption Duration Index
(SAIDI) and SAIFI, are not considered. The methodology is tested using a network with 20 components.

This paper presents a multiobjective methodology for the optimization of PM programs in EPDS focused on RCM. The proposed methodology differs from other references found in the literature in the following aspects: (1) the optimization model proposed is multiobjective and comprises real variables and decision binary variables. The first objective function evaluates the cost of maintenance actions and the second evaluates EPDS reliability over a considered planning horizon. The constraints considered in this model are: (i) the expected values of SAIFI and SAIDI service continuity indicators for each set of customers; (ii) the time (hours) availability of maintenance crews; and (iii) the limit of maintenance tasks for each type of equipment considering the complexity of each type of maintenance task. A dedicated Nondominated Sorting Genetic Algorithm (NSGA-II) is developed to solve the multiobjective optimization model [18]. (2) The reliability index of each piece of equipment is provided by a fuzzy inference system that considers: (i) the failure rates of the equipment provided by their probabilistic failure models; (ii) the set of factors (dynamics or static, such as age, operational effort, loading, time between maintenance tasks, operation number) that affects the functionality of the equipment; and (iii) maintenance tasks allocated over the considered planning horizon. (3) Each measure of $\mathrm{PM}$ is related to equipment reliability through the operation condition and equipment age. (4) The impact of PM programs on the reliability of the system under study is evaluated using an unreliability pseudo-index that considers the system reliability over the time for each sub-period of the planning horizon. This work considered a planning horizon of two years with monthly sub-periods of time.

The modelling was focused on the medium voltage components of an EPDS. The proposed methodology was tested in an EPDS of a company located in the state of São Paulo, Brazil. The distribution system consists of 733 components derived from three overhead, radial and independent feeders of $13.8 \mathrm{kV}$. The components considered are distribution transformers (TR), voltage regulators (VR), circuit breakers $(\mathrm{CB})$, capacitor banks $(\mathrm{CP})$, protection and maneuver switches (SWP), and primary cables (PC).

This work is composed of this introductory section on planning maintenance, with a focus on reliability, and the following sections. Section 3 presents a description and details about the optimization model and the methodologies for obtaining the reliability indices and performing the impact assessment of the maintenance tasks. Section 4 presents the solution technique used to solve the multiobjective mathematical model. Section 5 presents the results obtained from implementing the proposed methodology. Section 6 presents the contributions and conclusions about the proposed methodology.

\section{Mathematical model}

The mathematical model proposed in this paper is multiobjective, combinatorial, binary, dynamic, nonlinear and restricted and is given by Eqs. (1)-(13). The objective function $f_{1}$ represents the costs of scheduled maintenance actions, while $f_{2}$ considers a global pseudo-index of unreliability produced by the tasks' maintenance programs. This pseudo-index is the arithmetic mean of the unreliability partial index obtained for each monthly sub-interval of time, $t$, of an annual planning horizon $p h$. The pseudo-index obtained in $f_{2}$ is the average of the fault probabilities for each system section, weighted by the active power loads $w_{s}$ fed by section $s \in S$. The lower this value, the better the expected system reliability. This concept was used because the target of RCM is to improve the reliability index of equipments and consequently the reliability of all 
distribution system, but could be used the concept of not supplied energy to quantify this objective function. In function $f_{1}$, the cost of each $m$ maintenance action $\operatorname{cost}_{(e, m)}$ for each piece of analyzed equipment $e \in E$ is updated monthly through rate $j$.

Minimize $f(X)=\left\langle f_{1}(X), f_{2}(X)\right\rangle$

subject to,

$\sum_{m=2}^{\left|M_{\mathrm{tr}}\right|}\left[\operatorname{atc}_{\left(e_{\mathrm{tr}}, m\right)} \cdot x_{\left(e_{\mathrm{tr}}, m\right)}^{t}+\operatorname{artc}_{e_{\mathrm{tr}}} \cdot\left(1-C_{\text {cons }_{i}}(t)\right)\right]$

$\leq$ dic_month ${ }_{\min } \quad \forall t \in P H$ and $\forall e_{\mathrm{tr}} \in E_{\mathrm{TR}}$

$\sum_{t=1+3(q-1)}^{3 q}\left\{\sum_{m=2}^{\left|M_{\mathrm{tr}}\right|}\left[\operatorname{atc}_{\left(e_{\mathrm{tr}}, m\right)} \cdot x_{\left(e_{\mathrm{tr}}, m\right)}^{t}+\operatorname{artc}_{e_{\mathrm{tr}}} \cdot\left(1-C_{\text {cons }_{i}}(t)\right)\right]\right\}$
$\leq 1.5 \times$ Dic_trim $_{\min } \quad \forall q \in P H Q$ and $\forall e_{\mathrm{tr}} \in E_{\mathrm{TR}}$

$\sum_{t=1+12(y-1)}^{12 y}\left\{\sum_{m=2}^{\left|M_{\mathrm{tr}}\right|}\left[\operatorname{atc}_{\left(e_{\mathrm{tr}}, m\right)} \cdot x_{\left(e_{\mathrm{tr}}, m\right)}^{t}+\operatorname{artc}_{e_{\mathrm{tr}}} \cdot\left(1-C_{\text {cons }_{i}}(t)\right)\right]\right\}$

$\leq 3 \times$ Fic_year $_{\min } \forall y \in P H Y$ and $\forall e_{\mathrm{tr}} \in E_{\mathrm{TR}}$

$\sum_{m=2}^{\left|M_{\mathrm{tr}}\right|}\left[x_{\left(e_{\mathrm{tr}}, m\right)}^{t}+\left(1-C_{\text {cons }_{i}}(t)\right)\right] \leq$ fic_month $_{\min }$

$\sum_{t=1+3(q-1)}^{3 q}\left\{\sum_{m=2}^{\left|M_{t r}\right|}\left[x_{\left(e_{t r}, m\right)}^{t}+\left(1-C_{\text {cons }_{i}}(t)\right)\right]\right\}$

$\leq 1.5 \times$ fic_trim $_{\min } \quad \forall q \in P H Q$ and $\forall e_{t r} \in E_{T R}$

$\sum_{t=1+12(y-1)}^{12 y}\left\{\sum_{m=2}^{\left|M_{\mathrm{tr}}\right|}\left[x_{\left(e_{\mathrm{tr}}, m\right)}^{t}+\left(1-C_{\text {cons }_{i}}(t)\right)\right]\right\}$

$\leq 3 \times$ fic_year $_{\text {min }} \forall y \in P H Y$ and $\forall e_{t r} \in E_{T R}$

$\sum_{t=1}^{p h} a_{1} \cdot x_{(e, 2)}^{t}+a_{2} \cdot x_{(e, 3)}^{t}+a_{3} \cdot x_{(e, 4)}^{t} \leq b_{1} \quad \forall e \in\left\{E_{\mathrm{TR}} \cup E_{\mathrm{VR}} \cup E_{\mathrm{CB}}\right\}$

$\sum_{t=1}^{p h} a_{4} \cdot x_{(e, 2)}^{t}+a_{5} \cdot x_{(e, 3)}^{t} \leq b_{2} \quad \forall e \in\left\{E_{\mathrm{CP}} \cup E_{\mathrm{SWP}} \cup E_{\mathrm{PC}}\right\}$

$\sum_{e=1}^{\left|E_{f}\right|} \sum_{m=2}^{\left|M_{e}\right|}\left(\operatorname{atc}_{(e, m)}+\operatorname{atp}_{(e, m)}+\operatorname{atd}_{(e, m)}\right) \times x_{(e, m)}^{t} \leq t_{\text {feasible }_{f}}$

$\forall t \in P H$ and $\forall f \in F$

$\left[1-C_{e_{\mathrm{cp}}}(t)\right] \leq u r_{\max } \quad \forall e_{\mathrm{cp}} \in E_{\mathrm{CP}}$

$\sum_{m=1}^{\left|M_{e}\right|} x_{(e, m)}^{t}=1 \quad \forall t \in P H \quad$ and $\forall e \in E$

$x_{(e, m)}^{t} \forall t \in P H, \forall e \in E$ and $\forall m \in M_{e}$ where,

$f_{1}(X)=\sum_{t=1}^{p h} \sum_{e=1}^{|E|} \sum_{m=2}^{\left|M_{e}\right|} \operatorname{cost}_{(e, m)} \times(1+j)^{t} \times x_{(e, m)}^{t}$
$f_{2}(X)=\frac{1}{p h} \sum_{t=1}^{p h}\left[\frac{\sum_{s=1}^{|S|}\left[1-C_{s}(t)\right] \times w_{s}}{\sum_{s=1}^{|S|} w_{s}}\right]$
$C_{S}(t)=\prod_{i=1}^{n} C_{\text {cons }_{i}}(t)$
$C_{\text {cons }_{i}}(t)=C_{e_{\mathrm{tr}}}(t) \times \prod_{i \in M_{\mathrm{sec} \mid e_{\mathrm{tr}}}} C_{m s_{i}}(t)$
$C_{m s_{i}}(t)=C_{e_{\mathrm{sw}}}^{m s_{i}}(t) \times C_{E_{\mathrm{PC}}}^{m s_{i}}(t) \times\left(\prod_{e_{\mathrm{vr}} \in E_{\mathrm{VR} \mid m s_{i}}} C_{e_{\mathrm{vr}}}(t)\right.$

Constraints (2)-(7) consider the indices of the continuity of power supply service, DIC and FIC, for each set of system customers. These indices, used in Brazil, are directly related to the indices SAIDI and SAIFI, respectively, internationally used as indicators of continuity of power supply [19]. Constraints (8) and (9) restrict the number of PM tasks allowed for each component. The constants $a_{1}$, $a_{2}, a_{3}, b_{1}$ and $b_{2}$ are chosen based on the company investment policy for maintenance. Constraint (10) considers the time availability of each maintenance crew for each feeder $f \in F$ in each time period $t \in P H$. For each scheduled maintenance task, the average planning time, crew displacement driving time and task execution time of scheduled maintenance are considered. For maintenance actions that are performed offline, several days of execution time can correspond to the changeover time of faulty equipment. Constraint (11) considers the maximum acceptable probability threshold for capacitor banks. Because this type of equipment failure mostly does not interrupt the power supply, the reliability of such equipment does not affect the overall continuity reliability index of the system. Thus, the reliability of such equipment is handled separately as a constraint of PM programs. Constraint (12) establishes that only one of the $m \in M_{e}$ maintenance tasks should be chosen for each piece of equipment $e \in E$ in each time period $t$ of the planning horizon. The solution of this model is a set $X$ containing the maintenance plan for each piece of equipment $e \in E$ along the planning horizon.

\subsection{Evaluation of reliability indices}

The evaluation of equipment reliability takes an important role in this work. The methodology for assessing the reliability consists of two steps [16,17]. In the first step, the historical database of the system under study is analyzed and probabilistic fault models $P(t)$ for each component $e \in E$ are obtained using specific stochastic processes $[20,21]$. In the second step, factors such as equipment failures are identified and modelled through fuzzy sets. Then, a fuzzy inference system is modelled, taking into account failure factors (that depend on each piece of equipment) and the probability of equipment failure that was obtained in the first step. The system outputs are the reliability indices $C_{e}(t)$ of each piece of equipment $e \in E$ in each time period $t \in P H$.

Modeling of fuzzy inference system rules was carried out with the assistance of operators and engineers who had extensive experience in the area. More details about the fuzzy inference system can be found in $[16,17]$. The reliability index $C_{S}(t)$ in each section $s \in S$ in Eq. (16) is the probability that no interruption will occur in any set of customers connected to them. 


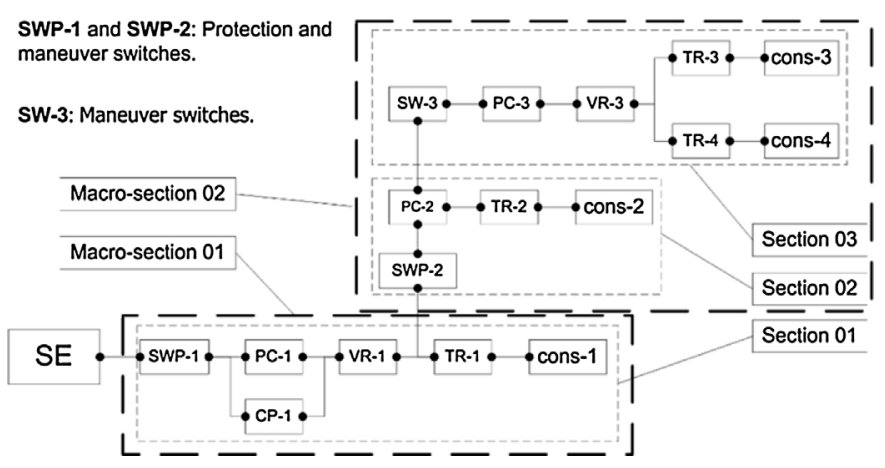

Fig. 1. Outline of a distribution network partitioned in sections.

A network section is a part of the network that can be isolated from the feeder by switching operations. Fig. 1 shows a distribution network with three sections defined by three switching devices and the formation of two macro sections. A set of sections protected by the same protection switch defines a network macro-section. The protection switches of distribution transformers are not considered in this work; only protection and maneuver switches are considered.

The reliability of each set of customers $C_{\text {cons }_{i}}(t)$ in Eq. (17) is calculated using the technique of network diagrams [5,24] and takes into account the reliability of the distribution transformer $C_{e_{\mathrm{tr}}}(t)$ and the reliability of each macro-section $C_{m s_{i}}(t)$ between this transformer and the substation. Specifically, the reliability of any set of customers in a section must take into account the probability of failure of all of the components present in that section, such as distribution transformers, network cables, voltage regulators, and switching devices, as well as the probability of component failures belonging to the remaining upstream sections of the macro-section.

\subsection{Update of reliability indices}

The reliability index $C_{e}(t)$ of each equipment $e \in E$ is a predictive index that takes into account the probability $P(t)$ of equipment failure and the operating conditions of the equipment, such as the age, operational effort, loading, time between maintenance tasks, and operation number. These factors depend on time and the maintenance tasks that are scheduled throughout the considered time interval [17]. Programming a level of maintenance activity $m$ to equipment $e$ in time $\bar{t} \in H P$ changes the expected values of the probability indices $P(t)$ and the expected conditions of $t$ for equipment failure factors for $t \geq \bar{t}$. Therefore, the fuzzy inference system should be updated, and the reliability index of equipment for all $t \geq \bar{t}$ should be re-evaluated. This feature makes the problem of maintenance task allocation a dynamic problem. Fig. 2 shows a flowchart for updating and evaluating reliability indices $C_{e}(t)$.

In this work, two to four types of maintenance tasks were adopted; these are defined depending on their structural complexity, namely: does not perform maintenance $\left(m_{1}\right)$; minimal maintenance $\left(m_{2}\right)$; average maintenance $\left(m_{3}\right)$; and complete maintenance $\left(m_{4}\right)$. Each $m$-level of each maintenance task allocated at time $t$ impacts the input data of the fuzzy inference system differently. Then, each variable (failure factor) is updated depending on the allocated maintenance task. Eq. (19) updates the variable age of each component $e$ at time $t$, depending on the real age of the component $a_{e}^{0}$ and the scheduled maintenance tasks. The restoration index $R_{(e, m)}^{t}$ is responsible for repositioning each device $e \in E$ on the timeline of the apparent lifetime (apparent age). Table 1 presents the values defined for the restoration index $R_{(e, m)}^{t}$ of distribution transformers considering a useful life period

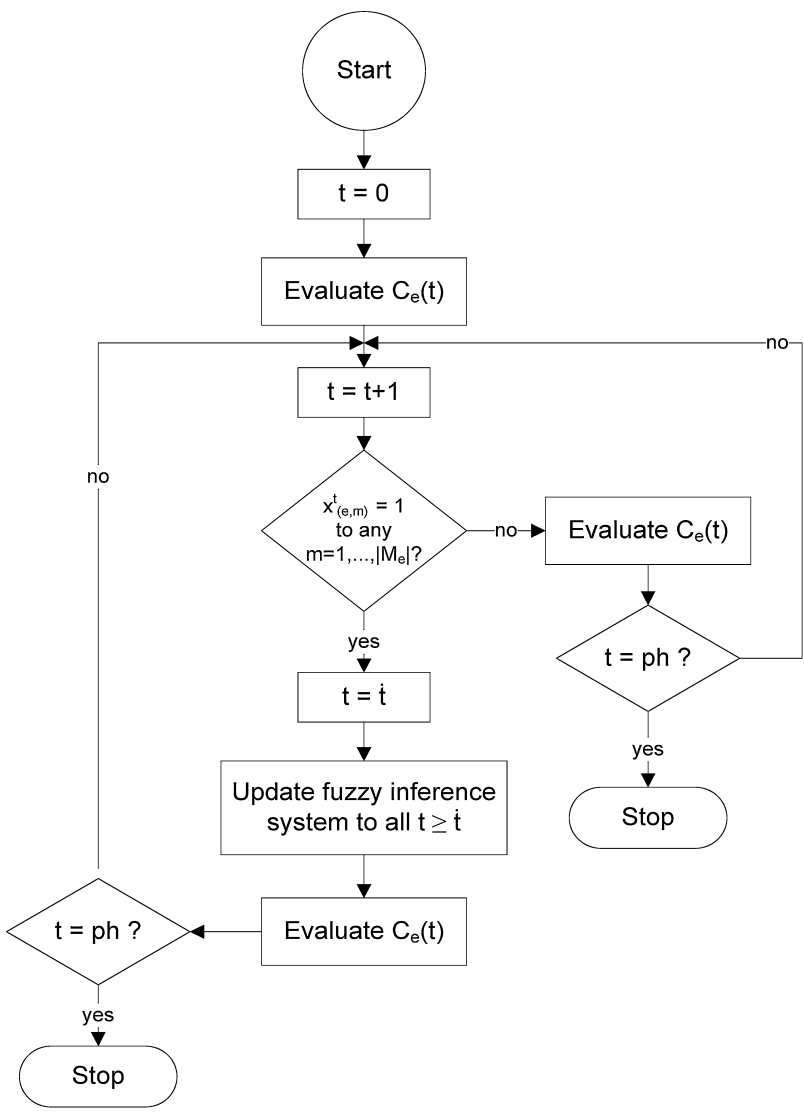

Fig. 2. Algorithm for evaluating and updating reliability indices $C_{e}(t)$.

of 20 years [23]. $R_{(e, m)}^{t}$ for the other equipment found in electrical distribution systems are described in [17].

$a_{e}^{t}=\left\{\begin{array}{c}a_{e}^{t-1}-\sum_{m=1}^{\left|M_{e}\right|}\left(x_{(e, m)}^{t} \times R_{(e, m)}^{t}\right), \quad \text { if } a_{e}^{t}>0 \\ 0, \quad \text { otherwise }\end{array}\right.$

For instance, the maintenance tasks defined for the PC components aim to restore the reliability of cross-arms and insulators of the allocated cables and not the cables themselves.

\section{Solution technique}

The methodology used to obtain the reliability index of equipments after each maintenance task makes the use of classical optimization techniques impracticable because of the amount and nature of the variables implicitly involved in the model.

Table 1

Indices of maintenance impact on distribution transformers.

\begin{tabular}{|c|c|c|c|c|c|c|c|c|c|}
\hline \multirow{2}{*}{$\begin{array}{l}\text { Age } \\
t \text { (year) }\end{array}$} & \multicolumn{4}{|c|}{$\boldsymbol{R}_{(e, m)}^{\boldsymbol{t}}($ year $)$} & \multirow{2}{*}{$\begin{array}{l}\text { Age } \\
t \text { (year) }\end{array}$} & \multicolumn{4}{|c|}{$\boldsymbol{R}_{(\boldsymbol{e}, \boldsymbol{m})}^{\boldsymbol{t}}(\mathrm{year})$} \\
\hline & $\boldsymbol{m}_{1}$ & $\boldsymbol{m}_{2}$ & $\boldsymbol{m}_{3}$ & $\boldsymbol{m}_{4}$ & & $\boldsymbol{m}_{1}$ & $\boldsymbol{m}_{2}$ & $\boldsymbol{m}_{3}$ & $\boldsymbol{m}_{4}$ \\
\hline 1 & 0 & 1 & 1 & 1 & 12 & 0 & 5 & 10 & 12 \\
\hline 2 & 0 & 2 & 2 & 2 & 13 & 0 & 5 & 10 & 13 \\
\hline 3 & 0 & 3 & 3 & 3 & 14 & 0 & 5 & 10 & 14 \\
\hline 4 & 0 & 4 & 4 & 4 & 15 & 0 & 5 & 10 & 15 \\
\hline 5 & 0 & 5 & 5 & 5 & 16 & 0 & 5 & 10 & 16 \\
\hline 6 & 0 & 5 & 6 & 6 & 17 & 0 & 5 & 10 & 17 \\
\hline 7 & 0 & 5 & 7 & 7 & 18 & 0 & 5 & 10 & 18 \\
\hline 8 & 0 & 5 & 8 & 8 & 19 & 0 & 5 & 10 & 19 \\
\hline 9 & 0 & 5 & 9 & 9 & 20 & 0 & 5 & 10 & 20 \\
\hline 10 & 0 & 5 & 10 & 10 & & & & & \\
\hline 11 & 0 & 5 & 10 & 11 & & & & & \\
\hline
\end{tabular}




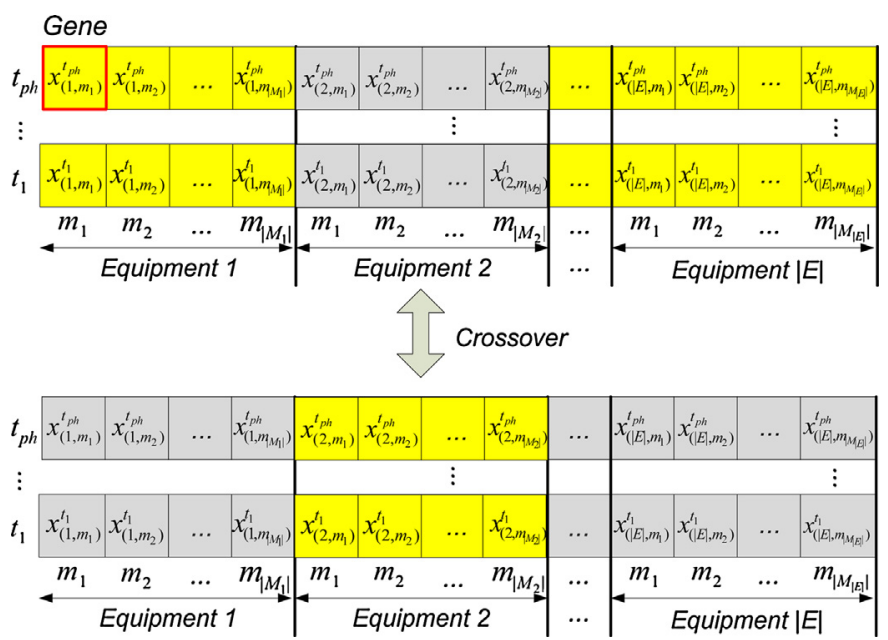

Fig. 3. Two chromosomes for NSGA-II considering $|E|$ equipment.

Some of them have a fuzzy nature, as described earlier. Thus, a multiobjective evolutionary algorithm, NSGA-II, was developed as the solution technique that allowed for a better fit to the model characteristics [25].

The NSGA-II proposed uses a dedicated codification system and specific mutation and recombination operators. In this algorithm, the number of genes in each chromosome is equal to the number of decision variables in the model. Specifically, each gene of the chromosome is a decision variable $x_{(e, m)}^{t}$. For each equipment, a gene chain represents the maintenance program along the planning horizon. In the recombination process, two cut off points are chosen to define the gene chain (maintenance programs) that will be interchanged to generate two new chromosomes. Fig. 3 illustrates this process, showing the chromosome representation.

The proposed mutation operator uses two steps of random selection. In the first step, one element of the chromosome is selected considering a constant mutation rate. In the second step, a gene of the maintenance program of the selected element in the previous step is selected randomly and then changed. Fig. 4 illustrates the mutation process considering element selected in the first step.

The penalty method [18] is used to address infeasible constraints. Classic methods of penalty use a high penalty factor to increase the objective function of solutions that violate the model constraints (minimization problems). In this paper, penalties calculated for violating the constraints (2)-(7) follow the rule applied by the regulatory agencies to calculate fines. These fines are applied in the form of compensation to be paid by utilities to customers who were affected by interruptions above the established limits. With this strategy, there are two goals: decreasing the penalty of solutions that violate constraints (2)-(7) and considering the payment of compensation as a planning strategy in the planning of PM.

\section{Results and discussion}

The proposed methodology was tested on an EPDS composed by three feeders referred by SYS3A. All of the feeders are considered
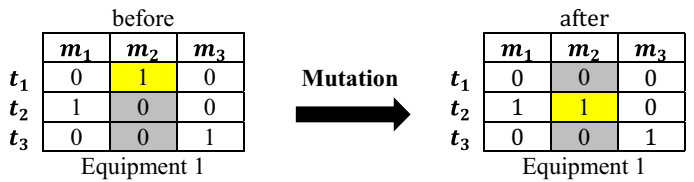

Fig. 4. Illustration of the mutation process for selected Equipment 1.
Table 2

Number of equipment pieces in each distribution feeder.

\begin{tabular}{lrcr}
\hline Feeder & P3-A1 & P3-A2 & P3-A3 \\
\hline Circuit breaker & 1 & 1 & 1 \\
Voltage regulator & 0 & 0 & 1 \\
Cables & 260 & 179 & 125 \\
Transformers & 42 & 32 & 22 \\
Switches & 23 & 19 & 26 \\
Capacitor banks & 0 & 0 & 1 \\
\hline
\end{tabular}

to be independent and they are feeding by the same substation P3. Table 2 presents the number of devices composing each of them.

The three feeders constitute an EPDS with 71 sections and supply energy to 96 sets of customers. Considering a planning horizon of 24 months and 733 components (Table 2), the optimization model is composed of 55,176 decision variables and 24,168 constraints. A set $X$ of PM programs that best represents the maintenance costs and reliability indices is expected as a result. The following subsections present the test results.

\subsection{Input data}

The evolutionary algorithm that is proposed as the solution requires making adjustments in the control parameters. These adjustments were performed through trial and error, and the best results obtained for these parameters are: number of the population's individuals, 800; mutation rate for the genes, 0.07 ; crossover rate, 0.9 ; number of individuals by tournament, 3; and stopping criterion, 50,000 generations. A database with the historical records of failures for 11 years was used to model the equipment reliability indices. For each piece of equipment in the same class, we adopted an equal cost for maintenance tasks of the same type, independent of model, power or other characteristics. Table 3 presents the costs $\cos t_{(e, m)}$, which are the mean values that were adopted in December 2013. The ratio dollar/euro in that period was $1 / 1.37$.

The constants appearing in the model, $a_{1}, a_{2}, a_{3}, a_{4}, a_{5}, b_{1}$ and $b_{2}$, are defined considering that only the maintenance activity on the high end, $m_{4}$, or at most two maintenance activities on the low end, $m_{2}$ and $m_{3}$, can be allocated to each piece of system equipment along the planning horizon that was considered. The values adopted for these constants are: $a_{1}=4, a_{2}=5, a_{3}=6, a_{4}=4, a_{5}=5$ and $b_{1}=b_{2}=9$.

The monthly time available, $t_{\text {feasible }_{f}}$, of each maintenance crew in hours for feeders P3-A1, P3-A2 and P3-A3 are 36.96, 18.48 and $18.48 \mathrm{~h}$, respectively. The limit values for the DIC and FIC interruption indices used in constrains (2)-(7) are considered to be equal for all feeders. The monthly, quarterly, and yearly DIC values in minutes are 116.64, 233.28 and 466.56, respectively, and the monthly, quarterly, and yearly FIC values in minutes are 1.524 , 3.008 and 6.018 , respectively. These values correspond to $15 \%$ of the limit set up by the regulatory agencies in Brazil. These percentages were adopted based on the results of studies regarding component

Table 3

Cost of maintenance tasks by equipment class.

\begin{tabular}{llrrr}
\hline \multirow{2}{*}{ System components } & \multicolumn{4}{l}{ Costs of maintenance tasks (US\$) } \\
\cline { 2 - 5 } & $m_{1}$ & $m_{2}$ & \multicolumn{1}{c}{$m_{3}$} & \multicolumn{1}{c}{$m_{4}$} \\
\hline Transformers & 0 & 120 & 300 & 1974.50 \\
Voltage regulators & 0 & 120 & 1,500 & 12,500 \\
Capacitor banks & 0 & 200 & 500 & - \\
Circuit breakers & 0 & 200 & 300 & 12,500 \\
Cables & 0 & 8 & 100 & - \\
Switches & 0 & 15 & 50 & - \\
Switches oil/vacuum/SF6 & 0 & 50 & 400 & - \\
\hline
\end{tabular}




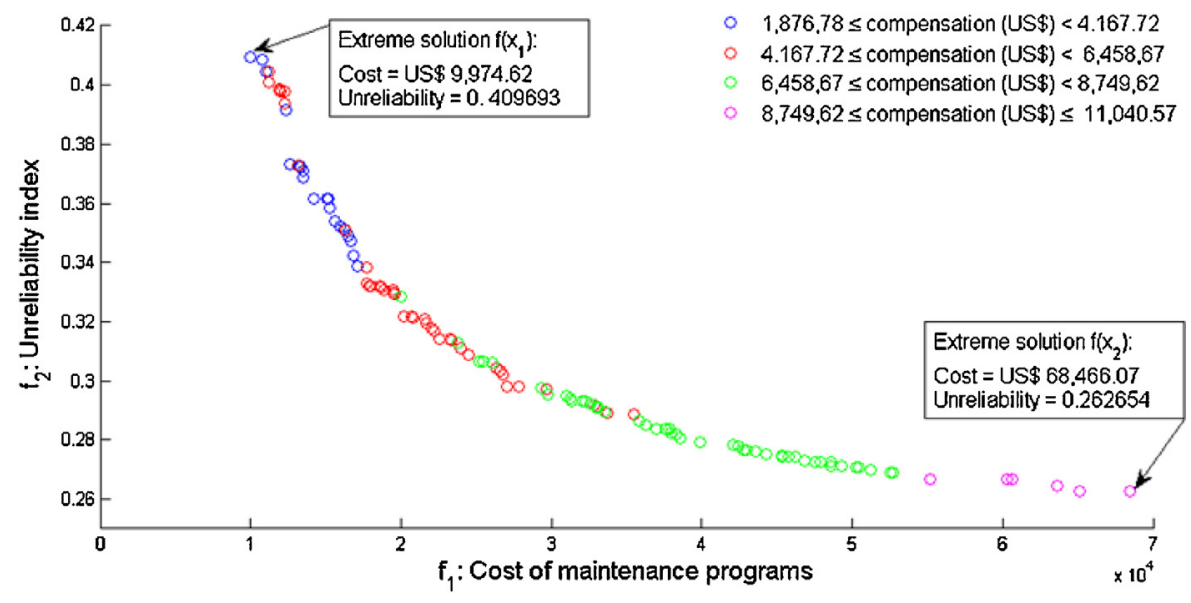

Fig. 5. Pareto Frontier of system SYS3A.

failures on overhead distribution systems [26]. The maximum supported value adopted for the failure probability (unreliability) $u r_{\max }$ of capacitor banks in equation (11) is 0.30 for all $e_{\mathrm{cb}} \in E_{\mathrm{CB}}$.

The values of the constants ate $(e, m), \operatorname{atp}_{(e, m)}$ and $\operatorname{atd}_{(e, m)}$ were extracted from the historical failure records of each equipment $e \in$ $E$ provided by the distribution company owner of the system under study. Other values utilized in fuzzy modelling of reliability indices can be found in [17]. In test 1, the proposed methodology is tested considering the penalization strategy described in Section 4 ; in test 2 , all constraints are treated equally through the penalty functions that use high values of the penalty parameters.

\subsection{Test 1}

The Pareto front obtained by testing the proposed methodology is composed of 115 non-dominated solutions, i.e., 115 equally important maintenance programs that balance the PM planning costs with the system reliability. Fig. 5 shows this frontier, along with two other highlighted solutions.

The costs of the maintenance programs include the costs of the compensation payment resulting from violations of constraints (2)-(7). These violations were already expected because of the relaxation of the penalization factor. All of the other constraints were fully satisfied. The Pareto solutions are classified according to the expected value of the compensation payment. It can be seen in Fig. 5 that these values are low, not exceeding, on average, US\$ 115.00 , with a compensation payment per set of customers considering the maintenance plan of the extreme solution $f\left(X_{2}\right)$ (greater cost).

The values of the compensation payments are generated by violating the limits of the continuity indices, depending on the number and execution time of maintenance in the distribution transformers. These values eventually exceed the limits set by constraints

Table 4

Number of maintenance tasks by section provided by $f\left(X_{2}\right)$ in Fig. 5 .

\begin{tabular}{|c|c|c|c|c|c|c|c|c|c|}
\hline \multirow[t]{2}{*}{ Sections } & \multicolumn{3}{|l|}{ P3-A1 } & \multicolumn{3}{|l|}{ P3-A2 } & \multicolumn{3}{|l|}{ P3-A3 } \\
\hline & Load (W) & PM & M.a/M.d (\%) & Load (W) & PM & M.a/M.d (\%) & Load (W) & PM & M.a/M.d (\%) \\
\hline 1 & $2,248,074$ & 4 & 100 & $1,741,870$ & 4 & 100 & 960,565 & 4 & 100 \\
\hline 2 & $2,248,074$ & 2 & 100 & $1,741,870$ & 2 & 100 & 960,565 & 2 & 100 \\
\hline 3 & $2,248,074$ & 32 & 100 & $1,741,870$ & 30 & 51.72 & 960,565 & 16 & 100 \\
\hline 4 & $67,918.66$ & 9 & 75 & $1,699,326$ & 56 & 79.50 & 960,565 & 8 & 100 \\
\hline 5 & $2,180,156$ & 48 & 100 & - & & & 235,908 & 1 & 3.85 \\
\hline 6 & 44,725 & 5 & 83.33 & - & & & 41,377 & 3 & 50 \\
\hline 7 & $1,360,349$ & 21 & 95.45 & 50,053 & 4 & 66.67 & 4729 & 4 & 66.67 \\
\hline 8 & 20,691 & 0 & 0 & - & & & 4729 & 9 & 90 \\
\hline 9 & $1,137,700$ & 61 & 98.38 & $1,266,298$ & 59 & 98.3333 & 54,197 & 8 & 66.67 \\
\hline 10 & 951,800 & 22 & 52.38 & - & & & 407 & 0 & 0 \\
\hline 11 & 79,852 & 10 & 100 & - & & & 724,656 & 36 & 52.94 \\
\hline 12 & 61,329 & 3 & 75 & 42,544 & 0 & 0 & 4398 & 1 & 50 \\
\hline 13 & 302,184 & 30 & 46.87 & 304,968 & 52 & 86.67 & 814 & 0 & 0 \\
\hline 14 & 276,708 & 20 & 100 & - & & & 814 & 0 & 0 \\
\hline 15 & 276,708 & 40 & 46.51 & 598,993 & 12 & 37.5 & 407 & 1 & 25 \\
\hline 16 & 64,087 & 3 & 75 & 303,903 & 1 & 2.63 & 407 & 2 & 100 \\
\hline 17 & 44,725 & 6 & 75 & 42,544 & 2 & 50 & 330,482 & 16 & 53.34 \\
\hline 18 & 44,725 & 6 & 75 & 200,793 & 19 & 43.18 & 463 & 7 & 87.50 \\
\hline 19 & 652,791 & 54 & 90 & 142,18 & 42 & 87.50 & 463 & 0 & 0 \\
\hline 20 & 345,508 & 37 & 92.50 & 42,544 & 2 & 50 & 1908 & 32 & 100 \\
\hline 21 & 192,099 & 2 & 33.33 & & & & 1373 & 4 & 66.67 \\
\hline 22 & 64,033 & 6 & 50 & & & & 535 & 1 & 50 \\
\hline 23 & 118,452 & 14 & 100 & & & & 535 & 0 & 0 \\
\hline 24 & 118,452 & 40 & 90.90 & & & & - & - & - \\
\hline 25 & & & & & & & 60,604 & 0 & 0 \\
\hline 26 & & & & & & & 407 & 3 & 75 \\
\hline 27 & & & & & & & 2779 & 1 & 16.67 \\
\hline Total & $2,248,074$ & 475 & 77.27 & $1,741,870$ & 285 & 66.28 & 960,565 & 159 & 50.31 \\
\hline
\end{tabular}


(3)-(8) when added to the expected values according to the failure probability of the network components. Three different aspects of the proposed methodology can explain this fact: (a) to improve the overall reliability index, the search methodology allocates periodic preventive maintenance for the distribution transformers, reducing the continuity indices of each set of customers ; (b) the limits of the continuity indicators (terms independent of the constraints (2)-(7)) consider only interruptions because of failures of system components, while the expected values for these indicators also consider interruptions because of the scheduled PM; and (c) the values of $a t e_{\left(e_{\mathrm{tr}}, m_{\mathrm{tr}}\right)}$ and arte $_{e_{\mathrm{tr}}}$ are relatively high compared to the limits of DIC and FIC in the customer set. Thus, PM programs that have greater reliability are more likely to violate constraints (2)-(7). Regardless, this result shows the importance of accurately calibrating these constraint limits.

The sections that feed larger loads receive a greater number of maintenance tasks proportionally to the limit of maintenance. It can be seen in Table 4 that the method seeks to allocate the maximum number of maintenance activities for the components of the sections fed a higher load. According to this table, the maintenance task numbers per feeder also follows the required demand.

Some discrepancies observed in Table 4, for example, in network sections 6 and 10, occur because of the difference in the number of cable sections. According to Eq. (18), the reliability of the cables has a significant influence on the composition of the reliability indices for the sets of customers. Consequently, the MOGA algorithm prioritizes the allocation of maintenance in this type of component in relation to others that have less influence. Table 5 shows this prioritization, mainly for program maintenance provided by the extreme solution $f\left(X_{2}\right)$.

It should be emphasized that the methodology has been programmed so that if the heuristic choice allocates maintenance activity for cables in one section, all of the cable parts in the section should receive maintenance activity. However, the greater the number of cable portions that receive maintenance, the higher the cost. Thus, the methodology balances the cable section number with the number of maintenance tasks allocated to these components. In addition, the methodology prioritizes the system reliability index as a whole as well as the total cost of the maintenance program, without prioritizing particular sections. In the specific case of network section 6 and 10, the methodology allocated two PMs for a single cable portion of the section and one
Table 5

Distribution of the number and type of allocated preventive maintenance.

\begin{tabular}{llrrr}
\hline Maintenance programs & Components & \multicolumn{3}{c}{ Maintenance task } \\
\cline { 3 - 5 } & & $\boldsymbol{m}_{2}$ & $\boldsymbol{m}_{3}$ & $\boldsymbol{m}_{4}$ \\
\hline Provided by extreme solution $f\left(X_{1}\right)$ & CB & 2 & 2 & 0 \\
& TR & 4 & 1 & 10 \\
& SWP & 4 & 11 & 0 \\
& VR & 1 & 0 & 0 \\
& CP & 1 & 0 & 0 \\
Total of allocated maintenance & PC & 103 & 21 & - \\
Provided by extreme solution $f\left(X_{2}\right)$ & $\mathbf{1 6 0}$ & & & \\
& CB & 4 & 2 & 0 \\
& TR & 33 & 23 & 8 \\
ThP & VR & 45 & 23 & 0 \\
& CP & 0 & 0 & 0 \\
& PC & 1 & 0 & 0 \\
& $\mathbf{9 1 9}$ & 559 & 221 & - \\
\hline
\end{tabular}

Table 6

Maintenance program of one section of the SYS3A system provided by the extreme solution $f\left(X_{2}\right)$.

\begin{tabular}{llll}
\hline Feeder-Section & Component & $\begin{array}{l}\text { Maintenance task } \\
\text { (type)-(month) }\end{array}$ & \\
\hline P3-A1-11 & SWP & $3-4$ & $2-24$ \\
& PC & $3-3$ & $2-24$ \\
& PC & $3-3$ & $2-24$ \\
& PC & $3-3$ & $2-24$ \\
\hline
\end{tabular}

PM to 37 parts of network section 10, resulting in the discrepancy presented in Table 4.

Maintenance programs can also be evaluated in terms of the reliability indices expected for the feeders. Fig. 6 shows these indices as a function of PM programs provided by the extreme solution $f\left(X_{2}\right)$ and by the program without any maintenance tasks scheduled. Fig. 7 shows the reliability indices expected for feeder P3-A1 as a function of the extreme solutions $f\left(X_{1}\right)$ and $f\left(X_{2}\right)$.

Table 6 shows a partial output of the proposed methodology to one section provided by the maintenance program of the extreme solution $f\left(X_{2}\right)$. The maintenance type, planning horizon month and maintenance quantity are shown for each piece of equipment.

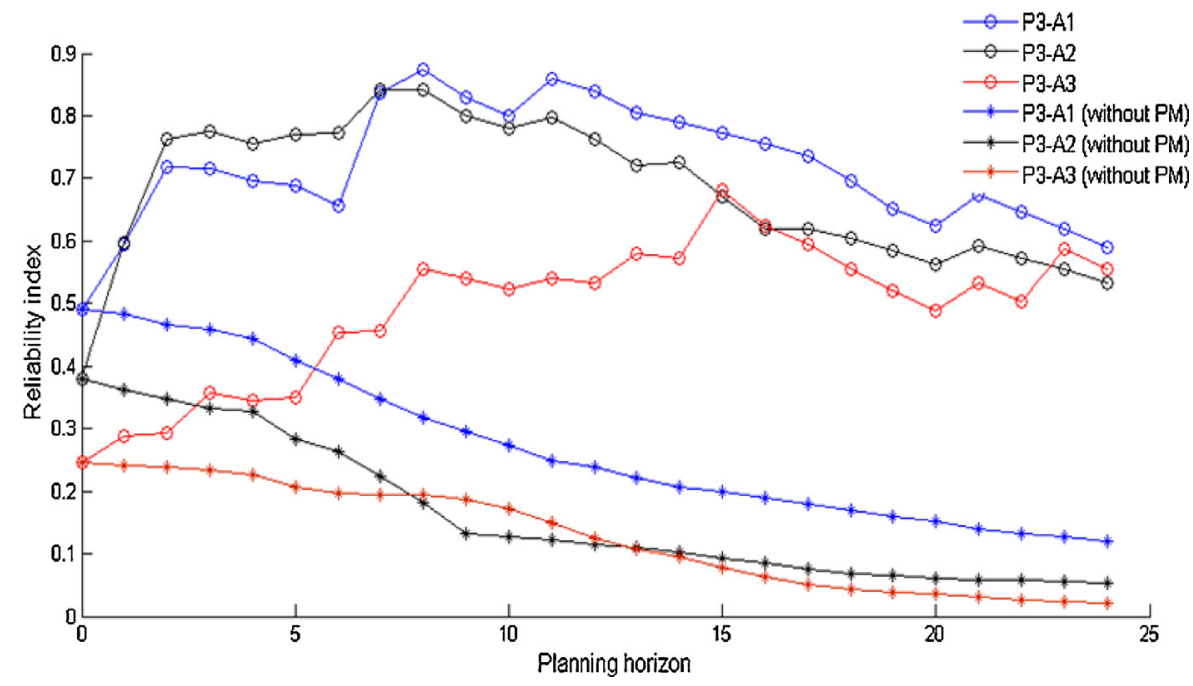

Fig. 6. Expected behavior for the feeder's reliability depending on the PM program of the extreme solution $f\left(X_{2}\right)$. 


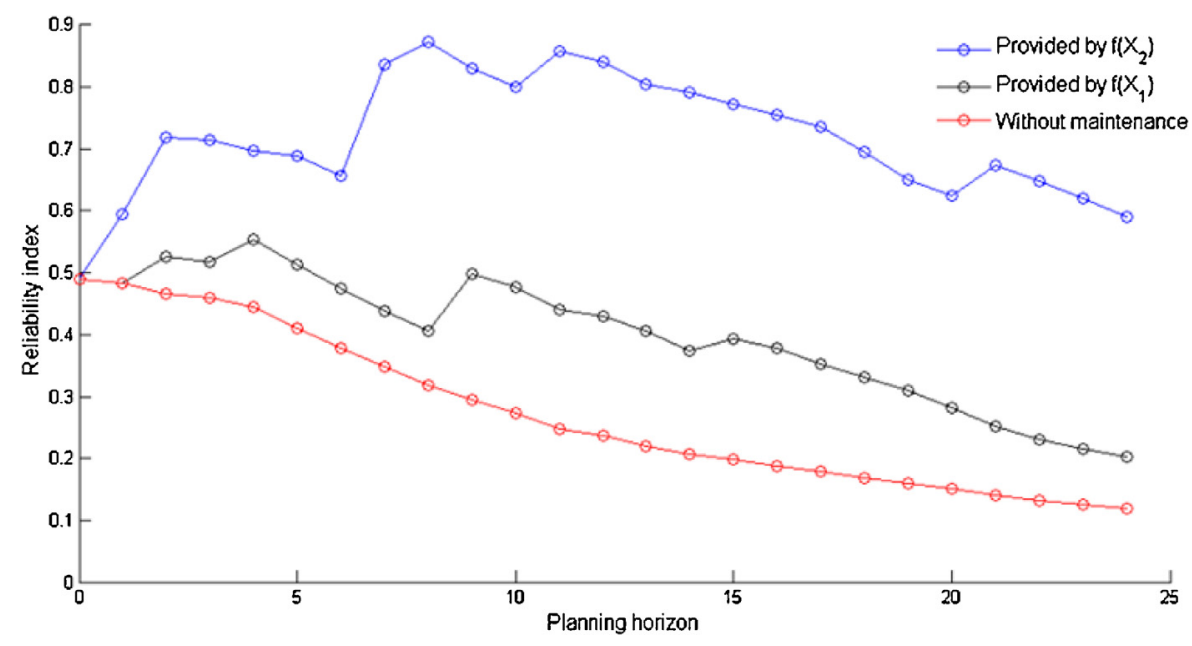

Fig. 7. Expected behavior for the P3-A1 feeder's reliability depending on three PM programs.

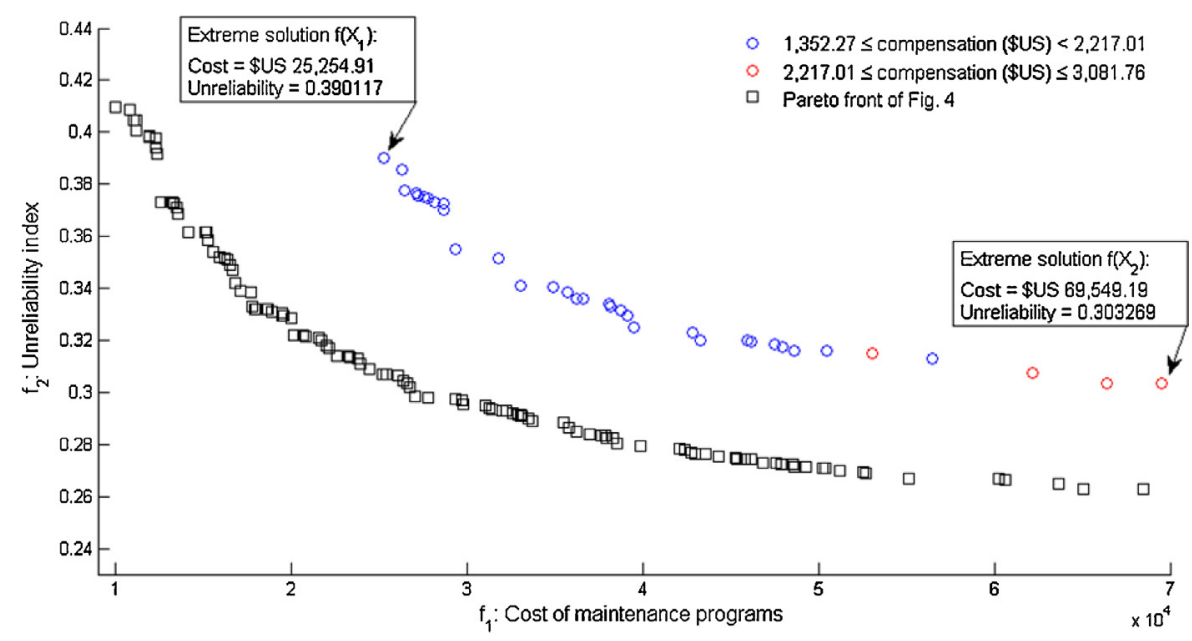

Fig. 8. Pareto curves of tests 1 (squares) and 2 (circles).

\subsection{Test 2}

Test 2 considers high levels of penalties factors on the violated constraints. All of the penalty functions have the same weighting factor. In this test, the Pareto front is composed of 35 nondominated solutions. Fig. 8 shows the Pareto frontiers obtained in the two tests, where it can be observed that the cost of compensation payments decreased. However, the unreliability index increased. The deterioration of the system reliability indices is also reflected on the reliability indices expected for all feeders.

Another important effect observed in test 2 is the reduction of the non-dominated solution number on the Pareto front.

\section{Conclusions}

The proposed methodology is able to obtain optimized solutions of good quality for preventive maintenance programs for EPDS. The versatility of this methodology allows the decision maker to obtain optimal maintenance plans for a system composed of several feeders. Moreover, it can also achieve optimal plans for each feeder independently, without having to change the optimization model.

The methodology for assessing the reliability of the components allows for the implementation of different factors that affect the component reliability, improving the reliability indices that were obtained. Although generalized costs are used (Table 3), the quality of the maintenance plan can be improved if specific maintenance costs for each component are considered. In addition, the quality of the component database also contributes to the solution quality.

The proposed methodology preferentially allocated maintenance tasks that have less impact on the reliability of the components, i.e., type $m_{2}$ and $m_{3}$ in tests 1 and 2 . These activities have the lowest maintenance cost and also have a smaller impact on the reliability of the equipment. However, the initial condition of the equipment makes it impossible to allocate higher impact maintenance tasks.

\section{References}

[1] J. Endrenyi, G. Anders, A.L. Silva, Probabilistic evaluation of the effect of maintenance on reliability: an application to power systems, IEEE Trans. Power Syst., Piscataway 13 (2) (1998) 576-583.

[2] L. Bertling, R. Allan, R. Eriksson, A reliability-centered asset maintenance method for assessing the impact of maintenance in power distribution systems, IEEE Trans. Power Syst., Piscataway 20 (1) (2005) 75-82.

[3] N. Phoothong, P. Vanittanakom, N. Teera-achariyakul, et al. Optimal preventive maintenance budget setting for electric power distribution utilities, Proceedings of the international conference on electrical engineering/eletronics, computer, telecommunications and information technology, Piscataway, 2008, pp. 957-960.

[4] A. Smith, Reliability-centered Maintenance, 1st ed., McGraw-Hill, Boston, 1993. 
[5] R. Brown, Electric Power Distribution Reliability, 2nd ed., CRC Press, New York, 2009.

[6] R. Brown, M. Marshall, Budget constrained planning to optimize power system reliability, IEEE Trans. Power Syst., Piscataway 15 (2) (2000) 887-892.

[7] F. Yang, C.S. Chang, Optimisation of maintenance schedules and extents for composite power system using multiple-objective evolutionary algorithm, IET generation, Transm. Distrib. 3 (10) (2009) 930-940.

[8] J. Heo, G. Park, Y.T. Yoon, et al. Optimal maintenance strategies for transmission systems using the genetic algorithm, Proceedings of IEEE PES transmission and distribution conference and exposition, New Orleans, 2010, pp. 1-6.

[9] A. Sittithumwat, F. Soudi, K. Tomsovic, Optimal allocation of distribution maintenance resources with limited information, Electric Power Syst. Res. 68 (2004) 208-220.

[10] P. Hilber, V. Miranda, M. Matos, et al., Multiobjective optimization applied to maintenance policy for electrical networks, IEEE Trans. Power Syst., Piscataway 22 (4) (2007) 1675-1682.

[11] P. Vanittanakom, N. Phoothong, T. Khatsaeng, et al., Reliability centered maintenance for pea power distribution systems, in: Proceedings of the international conference on electrical engineering, Okinawa, Japan, July, 2008.

[12] N. Teera-achariyakul, K. Chulakhum, D. Rerkpreedapong, et al. Optimal allocation of maintenance budgets for reliability target setting, Proceedings of the Asia Pacific power and energy engineering conference (APPEEC), Chengdu, 2010, pp. $1-4$.

[13] P. Dehghanian, M. Fotuhi-Firuzabad, F. Aminifar, et al., A comprehensive scheme for reliability centered maintenance in power distribution systems. Part I: methodology, IEEE Trans. Power Deliv., Piscataway 28 (2) (2013) 761-770.

[14] P. Dehghanian, M. Fotuhi-Firuzabad, F. Aminifar, et al., A comprehensive scheme for reliability-centered maintenance in power distribution systems. Part II: numerical analysis, IEEE Trans. Power Deliv., Piscataway 28 (2) (2013) 771-778.

[15] P. Hilber, Maintenance Optimization for Power Distribution Systems, KTH School of Electrical Engineering, Stockholm, Sweden, 2008 (PhD thesis).

[16] D. Piasson, A.A.P. Biscaro, J.R.S. Mantovani, et al. A proposal for reliability evaluation of components on electric power distribution system integrating probabilistic models and fuzzy inference systems, Proceedings of IEEE PES transmission and distribution: Latin America conference and exposition (T\&DLA), vol. 6, Montevideo, 2012, pp. 1-8.

[17] D. Piasson, Plans Optimization of Components Maintenance of Electric Power Distribution Systems Centered on Reliability, São Paulo State University, São Paulo, 2014 (PhD thesis).

[18] K. Deb, Multi-objective Optimization Using Evolutionary Algorithms, WileyInterscience series in systems and optimization series, John Wiley \& Sons, Chichester, 2001.

[19] Transmission and Distribution Committee, IEEE guide for electric power distribution reliability indices, Transmission and Distribution Committee Std. (Revision of IEEE Std. 1366-2003) May 2012.

[20] R. Billinton, An analytical approach to evaluate probability distributions associated with the reliability indices of electric distributions systems, IEEE Trans. Power Deliv., Piscataway 1 (3) (1986) 245-251.

[21] C.J. Zapata, S.C. Silva, H.I. Gonzalez, et al. Modeling the repair process of a power distribution system, Proceedings of IEEE PES transmission and distribution: Latin America conference and exposition (T\&D-LA), Bogota, 2008, pp. 1-6.

[22] J. Endrenyi, S. Aboresheid, R. Allan, et al., The present status of maintenance strategies and the impact of maintenance on reliability, IEEE Trans. Power Syst., Piscataway 16 (4) (2001) 638-646.

[23] ANEEL, Study of Economic Useful Life and Depreciation Rate, 1 and 2, Federa School of Engineering Itajuba \& Center for Studies in Natural Resources and Energy, Itajuba, 2000.

[24] D. Chowdhury, A. Koval, Power Distribution System Reliability: Practical Methods and Applications, John Wiley \& Sons, New Jersey, 2009.

[25] K. Deb, A. Pratap, S. Agarwal, et al., A fast and elitist multiobjective genetic algorithm: NSGA-II, IEEE Trans. Acoust. Speech Signal Process. Evol. Comput., Piscataway 6 (2) (2002) 182-197.

[26] S.V.C. Horton, W.F. Goldberg, The failure rates of overhead distribution system components, IEEE Power Eng. Soc.-Transm. Distrib. Conf., Dallas (1991) 713-717.

[27] D. Srinivasan, A.C. Liew, J.S.P. Chen, C.S. Chang, Intelligent maintenance scheduling of distribution system components with operation constraints, Electric Power Syst. Res. 26 (1993) 203-209. 